\title{
Intense Magnetic Fields and Low Temperature Research.*
}

By Sir Ernest Rutherford, O.M., P.R.S.

$\mathrm{E}$ XPERIENCE has shown that the encouragement of research by minor grants for special apparatus and material is in reasonable measure provided for by the Government grant to the Royal Society, supplemented from the Society's own research funds. The grants to individual investigators from such sources are usually small but suffice to assist materially important researches of a limited scope. The situation, however, is very different when we consider large scale investigations of a pioneering character, which may require considerable financial support extending over a period of years in order to provide the necessary apparatus and technical assistance to bring the investigation to a definite conclusion. Few of our universities or other scientific institutions are sufficiently well endowed to support large scale researches of this kind, even when the research appears of marked promise and when the idea and the man are forthcoming. In considering the best method of utilising the balance of the Society's present resources, the Council of the Royal Society has decided that it can best help the advance of science by assisting major researches of this character, and, after careful consideration, was impressed with the fundamental importance of the researches at present being carried on by Dr. $\mathrm{P}$. Kapitza, at Cambridge, and the need for continuing this work on a more permanent basis.

It may be helpful at this stage to give a brief history of the origin and development of the work on which Dr. Kapitza has been engaged for the past eight years. Trained as an electrical engineer, Dr. Kapitza was lecturer in physics in the Petrograd Polytechnical Institute from 1918 until 1921. In 1921 he came to England and commenced research work in the Cavendish Laboratory, Cambridge. In 1922 he began experiments to test the possibility of obtaining intense magnetic fields by sending very strong currents through a coil for such a short interval that the heating effect in the coil is restricted to a permissible value. With the assistance of a grant from the Department of Scientific and Industrial Research, special accumulators were constructed to give the necessary intense currents for a short interval of about $\frac{1}{\mathrm{~B} 0} \mathrm{sec}$. In this way, fields up to 200,000 gauss were obtained, and it was found practicable to carry out experiments by this method, for example, on the Zeeman effect and on the deflection of $\alpha$-particles.

In order to carry these experiments still further, it was necessary to have a method of obtaining currents still larger and more under control. For this purpose, a generator of special design was constructed which gives, on short circuit, a current of about 70,000 amperes. The heavy current from the generator is passed for about one-hundredth of a second through a coil and is then broken by means of a specially designed automatic break. The Department of Scientific and Industrial

* Excerpts from the presidential address to the Royal Society delivered at the anniversary meeting on Dec. 1.
Research gave a very substantial grant for the construction of this apparatus, while Sir William Pope kindly provided a temporary laboratory to install the plant and to carry out the experiments. In 1926 the laboratory was opened formally by the late Lord Balfour, then Lord President of the Council, who had throughout taken an active interest in promoting these large scale experiments. This pioneering investigation, which was carried out in connexion with the Cavendish Laboratory, was only made possible by the generous and bold support of the Department of Scientific and Industrial Research, which, up to the present, has defrayed the complete cost of the apparatus and of the subsequent investigations.

One of the chief difficulties in these experiments has been to construct a coil strong enough to withstand the enormous disrupting forces which arise when a large current is passed through it. A number of coils have been constructed which give magnetic fields of between 300,000 and 400,000 gauss over a volume of about 3 c.c. There appears to be no inherent difficulty why fields of the order of 1 million gauss should not be obtained, when called for, by this method. As the current through the coil only lasts for about $\frac{1}{100}$ sec., oscillograph methods are used to determine the strength of the current and magnetic field and to follow the changes in the properties of the material under investigation. There is no special difficulty in conducting experiments with these momentary fields. In fact, a single photograph, obtained in $\frac{1}{10}$ sec., may give a complete quantitative record of the magnetic effects produced in a material over a wide range of magnetic field.

The application of these new methods of producing intense magnetic fields opens up a wide field of research where all magnetic properties can be examined in fields ten to thirty times greater than those hitherto available by the use of electromagnets.

As soon as the apparatus was in working order, experiments were begun to investigate the change of resistance of crystals of bismuth in these intense magnetic fields from atmospheric temperature to that of liquid air. This was followed by an extensive investigation of the behaviour of a large number of metals under corresponding conditions. In general, it was found that the change of resistance was at first approximately proportional to the square of the magnetic field, but above a certain critical field, which varied from metal to metal, the change of resistance tended to become linear. On the basis of these new results, Dr. Kapitza has suggested a new way of looking at the phenomena which underlie the electrical conductivity of metals and its variation with temperature. Preliminary experiments have also been made on the action of these strong fields on the para magnetism and diama.gnetism of certain substances, while a new and sensitive apparatus has been constructed to study magnetostriction effects. An 
account of the apparatus and the experimental methods, together with the results of some of these investigations, has been published by Dr. Kapitza in the Proceedings of the Royal Society.

Magnetic phenomena are shown in their simplest form at very low temperatures when the complications due to the motion of the atoms and molecules are largely avoided. In order to obtain temperatures still lower than that of liquid air, a liquid hydrogen plant has been installed during the present year, and is now in working order. Preliminary arrangements have been made to install a liquid helium plant when this is required for the investigations.

The grant given by the Department of Scientific and Industrial. Research for carrving out these researches expires in a few years, while the labora. tory temporarily lent for the purpose of these experiments is now required by the Chemical Department of the University. The Department of Scientific and Industrial Research, by its broadminded and far-seeing action, has done a great service to science in thus supporting, through their initial stages, investigations having no obvious or immediate application in practice or industry. Their support for an indefinite period, however, could scarcely be part of the Department's policy. On the other hand, it appeared to the Council of the Royal Society that investigations of this kind, in which new fields of knowledge are being opened up by new methods, had a peculiarly strong claim for support from those funds which the Society was holding ready for the furtherance of fundamental researches in pure science.

The Council of the Royal Society, in addition to appointing Dr. Kapitza to a Messel professorship, has therefore agreed to offer the University of Cambridge the sum of $£ 15,000$ for the building of a suitable laboratory within the next three years, provided the University was prepared to offer an appropriate site and to defray the running expenses of the new laboratory. If the University of Cambridge concurs with these proposals, the Royal Society will thus have been instrumental in founding a new and up-to-date laboratory, primarily designed for carrying out researches in intense magnetic fields, but at the same time providing the essentials of a modern cryogenic laboratory for the study of magnetic and other effects at the lowest attainable temperatures.

The name of the new laboratory at Cambridge has not yet been settled, but it would be appropriate if it indicated the connexion with the Royal Society and with the late Dr. Iudwig Mond, whose bequest furnished the income from which the cost of the laboratory will be defrayed. It should be noted that among the purposes indicated in the will of Dr. Mond for the use of his bequest was " erecting new laboratories ".

It will be remembered that, thirty years ago, Great Britain was pre-eminent in the study of effects produced on matter by the low temperature produced with the aid of liquid hydrogen. It will be recalled that the late Sir James Dewar, with the technical assistance of Mr. Lennox, first produced liquid hydrogen in quantity in the laboratories of the Royal Institution in 1898, and in 1899 the first solid hydrogen was obtained. It was so early as 1893 that Dewar devised the vacuum flask which has proved to be of such fundamental importance in the technique of low temperatures and has so greatly simplified the handling of liquid gases. It is of interest to note that it was decided in 1902 to construct a liquid hydrogen plant, of capacity of about five litres of liquid hydrogen per hour, as a British Government exhibit to the St. Louis Exposition in 1904. This plant was placed in the competent hands of Mr. (now Sir) Joseph Petavel, and I well remember the interest of his demonstrations of the properties of liquid hydrogen at that Exhibition. Some time later, a small liquid hydrogen plant was installed by Dr. M. W. Travers, in the laboratory of the late Sir William Ramsay at University College, London.

In the meantime, an efficient cryogenic laboratory had been established at Leyden, under the direction of the late Prof. Kamerlingh Onnes. His success in liquefying helium and also the wide range and importance of the investigations carried out on the effects of low temperatures on the properties of matter are well known. It was only a few years ago that Prof. W. H. Keesom, who followed Onnes in the charge of this laboratory, was successful in producing solid helium.

A few years ago, owing to the energy and enthusiasm of Prof. J. C. McLennan, liquid hydrogen and helium plants were installed in the University of Toronto, and have proved their utility in a number of important researches. In recent years modern equipment for the liquefaction of hydrogen and helium has been installed in the Reichsanstalt, Berlin, by Dr. W. Meissner, and very valuable results have been already obtained. Dr. Franz Simon, of the University of Berlin, obtains the temperature of liquid helium by an ingenious method involving the use of liquid hydrogen and the absorption of helium gas by charcoal.

I am sure it will be gratifying to the Society to know that we may soon expect to have an up-todate cryogenic laboratory on a small scale in Great Britain. I believe that it is in helping such important schemes of research that the Society can best utilise any research funds which it already possesses or which may become available in the near future. It not infrequently happens that a promising line of research or the development of a new method may be held up or abandoned because of the difficulty of obtaining adequate financial support. In some important directions, advance can only be made with the help of technical assistance in the construction and use of special apparatus, in some cases on an almost engineering scale.

It is by the encouragement and support of such major researches, especially in their initial stages, that the Society can be of great service in helping the advance of fundamental science in Great Britain. Along such general lines, it is not difficult to foresee that the Society will exert an ever-increasing influence on the progress of science and thus promote still further the original intentions of its founders.

No. 3188, VoL. 126] 